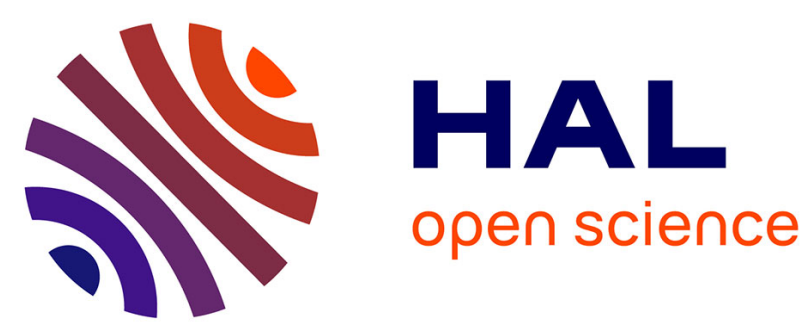

\title{
Energy-scalable temporal cleaning device for femtosecond laser pulses based on cross-polarized wave generation
}

\author{
Aurélien Ricci, Aurélie Jullien, Jean-Philippe Rousseau, Yi Liu, Aurélien \\ Houard, Patricia Ramirez, Dimitris N. Papadopoulos, Alain Pellegrina, \\ Patrick Georges, Frédéric Druon, et al.
}

\section{To cite this version:}

Aurélien Ricci, Aurélie Jullien, Jean-Philippe Rousseau, Yi Liu, Aurélien Houard, et al.. Energyscalable temporal cleaning device for femtosecond laser pulses based on cross-polarized wave generation. Review of Scientific Instruments, 2013, 84 (4), pp.043106. 10.1063/1.4801457 . hal-01303688

\section{HAL Id: hal-01303688 \\ https://hal-iogs.archives-ouvertes.fr/hal-01303688}

Submitted on 18 Apr 2016

HAL is a multi-disciplinary open access archive for the deposit and dissemination of scientific research documents, whether they are published or not. The documents may come from teaching and research institutions in France or abroad, or from public or private research centers.
L'archive ouverte pluridisciplinaire HAL, est destinée au dépôt et à la diffusion de documents scientifiques de niveau recherche, publiés ou non, émanant des établissements d'enseignement et de recherche français ou étrangers, des laboratoires publics ou privés. 


\section{AIP $\mid \begin{aligned} & \text { Review of } \\ & \text { Scientific Instruments }\end{aligned}$}

Energy-scalable temporal cleaning device for femtosecond laser pulses based on cross-polarized wave generation

Aurélien Ricci, Aurélie Jullien, Jean-Philippe Rousseau, Yi Liu, Aurélien Houard et al.

Citation: Rev. Sci. Instrum. 84, 043106 (2013); doi: 10.1063/1.4801457

View online: http://dx.doi.org/10.1063/1.4801457

View Table of Contents: http://rsi.aip.org/resource/1/RSINAK/v84/i4

Published by the American Institute of Physics.

Additional information on Rev. Sci. Instrum.

Journal Homepage: http://rsi.aip.org

Journal Information: http://rsi.aip.org/about/about_the_journal

Top downloads: http://rsi.aip.org/features/most_downloaded

Information for Authors: http://rsi.aip.org/authors






\title{
Energy-scalable temporal cleaning device for femtosecond laser pulses based on cross-polarized wave generation
}

\author{
Aurélien Ricci, ${ }^{1,2, a)}$ Aurélie Jullien, ${ }^{1}$ Jean-Philippe Rousseau, ${ }^{1}$ Yi Liu, ${ }^{1}$ Aurélien Houard, ${ }^{1}$ \\ Patricia Ramirez, ${ }^{3}$ Dimitris Papadopoulos, ${ }^{3,4}$ Alain Pellegrina, ${ }^{3,4}$ Patrick Georges, ${ }^{3}$ \\ Frédéric Druon, ${ }^{3}$ Nicolas Forget, ${ }^{5}$ and Rodrigo Lopez-Martens ${ }^{1}$ \\ ${ }^{1}$ Laboratoire d'Optique Appliquée, ENSTA-Paristech, Ecole Polytechnique, CNRS, 91761 Palaiseau, France \\ ${ }^{2}$ Thales Optronique SA, Laser Solutions Unit, 2 Avenue Gay-Lussac, 78995 Elancourt, France \\ ${ }^{3}$ Laboratoire Charles Fabry, Institut d'Optique, CNRS, Univ Paris Sud 2, Avenue Augustin Fresnel, \\ 91127 Palaiseau Cedex, France \\ ${ }^{4}$ Laboratoire d'Utilisation des Lasers Intenses, CNRS, Ecole Polytechnique, CEA, Univ Pierre et Marie Curie, \\ Palaiseau, France \\ ${ }^{5}$ Fastlite, Centre scientifique d'Orsay - Bat. 503, Plateau du Moulon, 91401 Orsay, France
}

(Received 20 December 2012; accepted 25 March 2013; published online 17 April 2013)

\begin{abstract}
We report on a compact energy-scalable device for generating high-fidelity femtosecond laser pulses based on spatial filtering through a hollow-core fiber followed by a nonlinear crystal for crosspolarized wave (XPW) generation. This versatile device is suited for temporal pulse cleaning over a wide range of input energies (from 0.1 to $>10 \mathrm{~mJ}$ ) and is successfully qualified on different ultrafast laser systems. Full characterization of the XPW output is presented. In particular, we demonstrate the generation of $1.6 \mathrm{~mJ}$ energy pulses starting from $11 \mathrm{~mJ}$ input pulse energy. The temporal contrast of the pulses is enhanced by more than 4 orders of magnitude. In addition, pulse shortening from 40 fs down to 15 fs Fourier-transform limit yields an overall peak-power transmission of up to $50 \%$. This device not only serves as an integrated pulse contrast filter inside an ultrafast laser amplifier but also as a simple back-end solution for temporal post-compression of amplified pulses. () 2013 AIP Publishing LLC. [http://dx.doi.org/10.1063/1.4801457]
\end{abstract}

\section{INTRODUCTION}

Improving the temporal contrast of intense femtosecond laser pulses is the key to accessing higher intensities during laser-plasma interactions on solid targets. ${ }^{1-5}$ A common distinction is made between the incoherent contrast related to the amplified spontaneous emission (ASE) in the different amplification stages (ps- to ns-scale) and the coherent contrast close to the pulse peak (sub-ps-scale) related to the fidelity of pulse compression. Standard chirped pulse amplification (CPA) lasers provide an ASE contrast ratio between $10^{6}$ and $10^{8}$ when no dedicated pulse cleaning device is implemented. While electro-optic methods can remove pedestals and prepulses on the ns scale, several optical techniques can be combined to achieve contrast ratios above $10^{12}$ on a ps scale, such as saturable absorbers, ${ }^{6}$ second-harmonic generation, ${ }^{7,8}$ nonlinear Sagnac interferometers, ${ }^{9}$ optical parametric amplification (OPA) ${ }^{10-13}$ plasma mirrors, ${ }^{14-16}$ and cross-polarized wave (XPW) generation. ${ }^{17}$

Nowadays, OPA, PM, and XPW are the most widely used. In an OPA, the contrast is dominated by the parametric fluorescence due to the pump pulse. By using picosecond duration pump, contrast as high as $10^{10}$ can be achieved at a few tens of ps from the main peak in pre-amplifier stages. However, OPA represents a major shift in laser technology from conventional CPA and its implementation in a pre-existing laser chain comes at the expense of a complete modification

\footnotetext{
a) Author to whom correspondence should be addressed. Electronic mail: aurelien.ricci@ensta-paristech.fr
}

of its architecture. The second option is a plasma mirror (PM), which relies on the change of reflectivity of a dielectric surface as electrons are heated up into a plasma at light intensities of the order of $10^{16} \mathrm{~W} / \mathrm{cm}^{2}$. The dielectric surface initially transmits the incident laser radiation until the electron density becomes critical, which triggers the PM, i.e., the surface of the plasma reflects the remainder of the laser pulse. This technique can therefore be used directly at the output of the CPA chain to efficiently suppress laser radiation up to a few picoseconds before the pulse peak. A double-PM can achieve an increase in contrast ratio of up to 4 orders of magnitude with an energy transmission of 50\%. Because it is destructive, this technique requires the dielectric surface to be continuously refreshed between laser shots and generates a large amount of debris. Its implementation at high repetition rate quickly becomes tricky as the positioning of the target surface must be done with high accuracy at high speed. ${ }^{18}$

In this review, we are interested in the third option that is XPW. It is a degenerate four-wave mixing process relying on the anisotropy of the real part of the crystal thirdorder nonlinearity tensor $\chi^{(3)}$, coined as $\sigma$. The influence of $\chi^{(3)}$-anisotropy on changes of polarization state in crystals was first investigated some 25 years ago by Zheludev and co-workers, who called it "self-induced optical activity." 19,20 Theoretical investigations were then performed by Hutchings and co-workers. ${ }^{21,22}$ The term "XPW" came years later following early experimental and theoretical work from Saltiel and co-workers. ${ }^{23-25}$

XPW generation takes place in an isotropic crystal when an intense linearly polarized incident wave generates a new 
wave at the same wavelength but linearly polarized in the orthogonal direction. XPW can therefore be discriminated by using two crossed polarizers. Hence, the less intense parts of the pulses, such as pre- and post-pulses along with the incoherent pedestal, remain unconverted and are therefore rejected by the filter, leading to enhanced pulse contrast. In practice, this enhancement is limited by the typical extinction ratio of the crossed polarizers to approximately 4 orders of magnitude. Furthermore, the process is automatically phase-matched and achromatic over a broad spectral bandwidth, thus particularly suited for femtosecond laser pulses. A typical material used for XPW generation is Barium Fluoride $\left(\mathrm{BaF}_{2}\right)$ crystal with $\mathrm{z}([001])$ or holographic $([011])$ crystallographic orientation. Theory predicts a maximum XPW energy conversion efficiency around $35 \%$ when using [011]-cut $\mathrm{BaF}_{2}$ crystal with a concomitant pulse shortening factor of $\sqrt{3}$ corresponding to a pure third-order nonlinear process. ${ }^{26}$ In this case, the maximum peak-power transmission is of the order of $60 \%$. Requiring moderate intensity $\left(10^{12} \mathrm{~W} / \mathrm{m}^{2}\right)$, XPW filtering is usually implemented in a double-CPA scheme ${ }^{27}$ and is commonly used to improve the contrast of TW- and PWclass lasers. ${ }^{5,28-31}$ Commercial set-ups are currently provided by Thales Optronique $\mathrm{SA}^{32}$ and Amplitude Technologies. ${ }^{33}$

Recently, accurate control of the input spectral phase has highlighted the possibility of using the XPW process to smooth spectral amplitude and phase modulations and broaden the input laser spectrum beyond the $\sqrt{3}$ approximation, ${ }^{34,35}$ leading to even sharper temporal pulse profiles. This feature calls for new applications of XPW generation, such as spectral smoothing of few-cycle pulses, ${ }^{30,36}$ temporal pulse characterization, ${ }^{37,38}$ and temporal shaping of high-repetition rate fiber lasers. ${ }^{39}$

The main issue with XPW so far has been its limited ability to temporally clean multi-mJ energy pulses with high conversion efficiency. The typical intensity for XPW generation is limited by supercontinuum generation in the crystal. Because the spatial quality of the incident laser beam on the crystal is of foremost importance for optimal conversion, the crystal was originally positioned at the focus of a lens or mirror. However, for practical reasons, this scheme was limited to input energies of the order of a hundred $\mu \mathrm{J}$. A successful way to increase the XPW conversion efficiency was then to use a two-crystal scheme with thin crystals $(<2 \mathrm{~mm})$ at a given distance from one another..$^{40}$ In this case, the induced Gouy phase-shift due to self-focusing in the first crystal yields the appropriate phase-matching in the next crystal to achieve overall efficiencies of $20 \%-25 \%$ with [011]-orientated $\mathrm{BaF}_{2}$ crystals. However, the separation between the crystals quickly scales up with input energy, thus limiting the input energy into the set-up to a few hundreds of $\mu \mathrm{J}$. To efficiently filter higher energy pulses, efforts were made to improve the spatial quality of the input beam out of focus with a single crystal setup. This approach was implemented using a nonlinear technique for flat-top pulse shaping combined with spatial filtering through a hard aperture. ${ }^{35}$ Conversions efficiencies were good for sub-mJ input energies but scaling to higher energies has not yet been demonstrated. The use of spatial filtering through a hard aperture alone has been tested at the multimJ-level but yields limited XPW efficiency $(<10 \%){ }^{41}$

We recently proposed a new XPW set-up design based on spatial filtering through a hollow-core fiber (HCF) waveguide used in combination with a single-crystal scheme. Waveguiding of laser pulse in $\mathrm{HCF}$ with diameters much larger than the laser wavelength was first proposed by Marcatili and Schmeltzer. ${ }^{42}$ Some 15 years ago, Nisoli et al. proposed the use of a rare gas-filled $\mathrm{HCF}$ to nonlinearly compress ultrashort pulses. ${ }^{43,44}$ Here, the HCF provides accurate control of the beam spatial quality, producing filtered incident laser pulses with Gaussian divergence. With this scheme, internal efficiencies of $33 \%$ for [011]-oriented $\mathrm{BaF}_{2}$ crystals, close to the theoretical limit, were achieved. Moreover, we demonstrated the energy scalability of this technique in the range $100 \mu \mathrm{J}-$ $3.3 \mathrm{~mJ} .{ }^{45}$ In line with this previous publication, we present here in details the design of this scheme in the range $0.1 \mathrm{~mJ}-$ $11 \mathrm{~mJ}$. Beyond energy scalability, its performances in terms of contrast enhancement, spatial and spectral quality, and peak power transmission are assessed. In addition, we show that the XPW process can routinely shorten the pulses by a factor greater than 2. Hence, the generation of $1.6 \mathrm{~mJ}$ XPW pulses, with global throughput on the order of $20 \%$ and pulse shortening by a factor of up to 2.5 , leads to peak-power transmission of the proposed setup of 50\%. At this energy level, implementing a XPW filter at the end of the laser chain becomes of interest as high-field experiments can be performed directly after the filter.

\section{EXPERIMENTAL SET-UP}

The schematic of the proposed XPW set-up is shown in Fig. 1. The nonlinear crystal is placed between two crossed Glan polarizers. The first polarizer ensures that the input beam is perfectly linearly polarized. The second polarizer is used to discriminate the cross-polarized wave from the fundamental wave. This configuration prevents the transmission through the XPW filter of any crossed polarized component of the fundamental wave, which could degrade the output temporal contrast. The extinction ratio of the two polarizers limits in practice the contrast enhancement to maximum four or

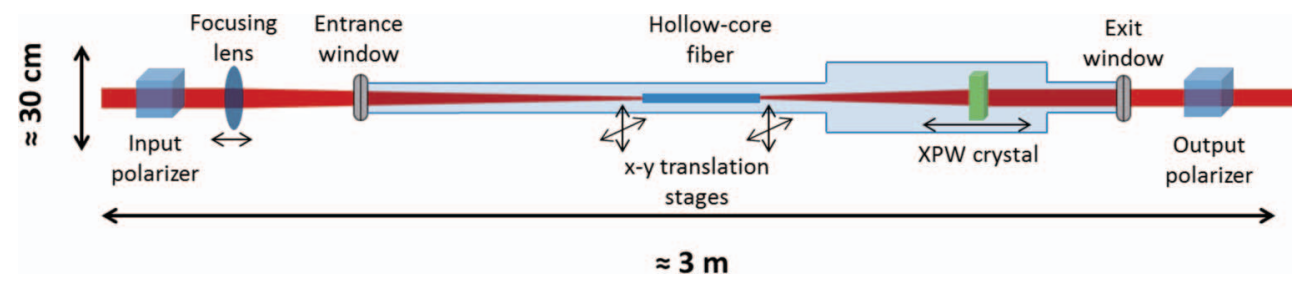

FIG. 1. Waveguide XPW set-up. 
five orders of magnitude. The proposed novel design consists of a vacuum chamber including a short HCF to spatially filter the beam before the crystal. The use of a HCF has several advantages. The output spatial beam profile is nearly perfectly Gaussian and propagates smoothly after the fiber (see Sec. III). Thus, working out of focus is now possible and yields conversion efficiencies close to the theoretical limit. Moreover, the beam is divergent after the fiber. Therefore, the laser input energy is no longer an issue as the crystal position can be easily shifted back and forth to match the optimum intensity. Two kinds of $250 \mu \mathrm{m}$ inner diameter HCF were tested during the experiments $(20 \mathrm{~cm}$ long, thick silica tube and $43 \mathrm{~cm}$ long, $1.7 \mathrm{~mm}$ outer diameter silica fiber from Femtolasers $\mathrm{GmbH}$ ) without noticeable change. Fiber alignment via $\mathrm{x}-\mathrm{y}$ micrometric translation stages is made independently of other vacuum parts by the use of flexible connectors.

The main requirements of this set-up are adequate coupling of the input beam into the fiber and its alignment. When correctly done, fiber transmission efficiency reaches more than $70 \%$. In order to optimally couple a laser beam into the fundamental mode of a fiber, it has to be focused down to a diameter size at $1 / e^{2}$ equal to 0.64 times the size of the inner core diameter of the fiber. ${ }^{46}$ In all the experimental situations shown below, the latter is $250 \mu \mathrm{m}$ which means that the optimal focus spot size is close to $160 \mu \mathrm{m}$. The next critical point consists in positioning the crystal to optimize XPW conversion efficiency while keeping intensity below the damage threshold of the crystal. Equation (1) below is used to estimate the distance needed after the fiber output in the range of energies we planned to use. It gives the relation between the diameter at $1 / e^{2}$ of the beam on the crystal, $\phi_{1 / e^{2}}^{\text {crystal }}$, and the distance from the fiber output to the crystal position, $D_{\text {fiber }}^{\text {crystal }}$ for a given Gaussian mode-field radius of the fiber, $w$. Experimentally, we measured the beam propagation after the fiber at low energy, in air. We found that its divergence corresponds with good agreement to the divergence of a Gaussian beam of waist $w=96 \mu \mathrm{m}$ (see Fig. 6, in Sec. III C),

$$
\phi_{1 / e^{2}}^{\text {crystal }}=1.22 \lambda \frac{D_{\text {fiber }}^{\text {crystal }}}{2 w} .
$$

Knowing the laser energy and pulse duration, one can estimate the minimum spot size on crystal and thus the minimum distance from the fiber end. The crystal is placed in a small vacuum chamber sealed by an acrylic glass window for direct user-control. An automated translation stage remotely controlled allows crystal position adjustment. The last issue that has to be addressed is minimizing nonlinear effects that may occur in glass windows and polarizers. To do so, we use ultra-thin AR-coated $500 \mu \mathrm{m}$ windows at both ends of the setup. The output polarizer may become an issue since we noticed that the beam is very slowly diverging after the crystal. An easy solution is to increase the beam size before propagation through the output polarizer. The typical size of the set-up is $3 \mathrm{~m}$ long and $30 \mathrm{~cm}$ wide. To demonstrate the remarkable energy scalability of the set-up, it has been tested on four different laser sources with energy ranging from $100 \mu \mathrm{J}$ to $11 \mathrm{~mJ}$ and duration comprised between $25 \mathrm{fs}$ and $50 \mathrm{fs}$, with similar
TABLE I. Characteristics of the laser sources used for the experiment. Central wavelength is around $800 \mathrm{~nm}$.

\begin{tabular}{|c|c|c|c|c|c|}
\hline $\begin{array}{l}\text { Laser } \\
\text { source }\end{array}$ & $\begin{array}{c}\text { Duration } \\
\text { (fs) }\end{array}$ & $\begin{array}{c}\text { Energy } \\
(\mathrm{mJ})\end{array}$ & $\begin{array}{l}\text { Rep. } \\
\text { rate }\end{array}$ & $\begin{array}{c}D_{\text {fiber }}^{\text {crystal }} \\
(\mathrm{cm})\end{array}$ & $\begin{array}{c}\phi_{1 / e^{2}}^{\text {crystal }} \\
(\mathrm{mm})\end{array}$ \\
\hline Fastlite (1) & 50 & 0.4 & $100 \mathrm{~Hz}$ & 10 & 0.8 \\
\hline $\operatorname{LCF}(2)^{45}$ & 25 & 1.5 & $1 \mathrm{kHz}$ & 25 & 1.5 \\
\hline LOA1 $(3)^{47}$ & 30 & 3.3 & $1 \mathrm{kHz}$ & 41 & 2.7 \\
\hline LOA2 (4) & 50 & 11 & $100 \mathrm{~Hz}$ & 56 & 3.5 \\
\hline
\end{tabular}

Thales Alpha 100

achievements. The characteristics of these laser sources are summarized in Table I.

In each experiment, the laser pulse was focused at the entrance of the fiber by a lens or a mirror placed on a translation stage to optimize the coupling efficiency (varying from $65 \%$ and $80 \%$ depending on the beam and fiber quality). The crystal position in each case is indicated in Table I along with the corresponding beam size on the crystal: between $10 \mathrm{~cm}$ and $56 \mathrm{~cm}$ after the output of the fiber to accommodate almost two orders of magnitude range in the input energy. The intensity on the [011] $2.5 \mathrm{~mm}$ crystal is nearly constant (taking into account HCF coupling losses) to a few $10^{12} \mathrm{~W} / \mathrm{cm}^{2}$. Our results show the flexibility of this scheme and how the same set-up can be used for input energies ranging from several hundred of microjoules up to more than $10 \mathrm{~mJ}$. Besides contrast enhancement, two important features have been achieved in all these cases : near-optimum XPW efficiency (30\%) with a single-crystal scheme leading to a standard global transmission of $20 \%$ and significant spectral broadening and shaping.

\section{RESULTS AND DISCUSSION}

\section{A. Energy-scalable single-crystal scheme and conversion efficiency}

Efficiency curves for the different laser sources as a function of input energies are shown on a logarithmic scale in Fig. 2. In each case, curves exhibit a smooth slope until the saturation regime is reached and efficiencies around $30 \%$ are achieved. In dotted line, we plotted the theoretical efficiency

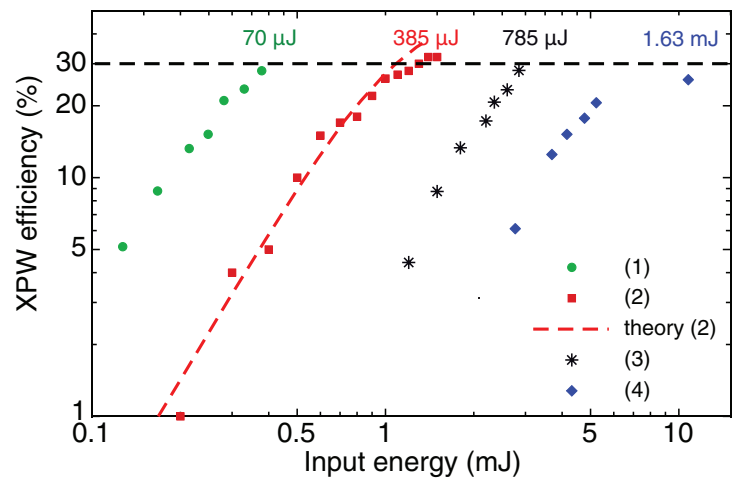

FIG. 2. Internal XPW efficiencies as a function of the input energies for the four different laser sources used. Theoretical curve in the case of laser (2) was obtained with a home-made simulation code. Maximum XPW output energy for each laser is shown on top of the plots. Fiber transmission and reflection losses are taken into account. 
TABLE II. XPW filter performances for the different laser sources used. Energy throughput represents the global transmission of the set-up. Spectral broadening is defined as the ratio of the full-width at half-maximum (FWHM) of the XPW spectrum over the FWHM of the incident laser spectrum.

\begin{tabular}{lcccc}
\hline \hline $\begin{array}{c}\text { Laser } \\
\text { source }\end{array}$ & $\begin{array}{c}E_{\text {in }} \\
(\mathrm{mJ})\end{array}$ & $\begin{array}{c}\text { Energy } \\
\text { throughput (\%) }\end{array}$ & $\begin{array}{c}\text { Spectral } \\
\text { broadening }\end{array}$ & $\begin{array}{c}\text { Contrast } \\
\text { improvement }\end{array}$ \\
\hline Fastlite (1) & 0.4 & 17.3 & 2.5 & $10^{4 \mathrm{a}, \mathrm{b}}$ \\
LCF (2) & 1.5 & 25.6 & 2.3 & $10^{4 \mathrm{a}}$ \\
LOA1 (3) & 3.3 & 23.8 & 2.4 & $10^{4 \mathrm{a}}$ \\
LOA2 (4) & 11 & 14.8 & 2.7 & $10^{4-5}$ \\
\hline \hline
\end{tabular}

${ }^{\mathrm{a}}$ Measured extinction ratio of the polarizers (contrast itself was not measured).

${ }^{\mathrm{b}}$ Coherent contrast was measured at $10^{2}-10^{3}$ between 100 and $400 \mathrm{fs}$ before the main peak (see Fig. 4)

calculated with a home-made MATLAB code in the case of the LCF laser source (2). It demonstrates the good agreement between the theoretical predictions and the observed efficiencies up to $30 \%$. This is a direct consequence of the spatial filtering through the fiber which optimizes the spatial profile (Gaussian and smooth) on the crystal. Furthermore, it mitigates Kerr focusing in the crystal by seeding it with a divergent beam (see Subsection III C). The consequent nonlinear phase mismatching is reduced and the conversion of the XPW signal is optimized even for long crystal length $(>3 \mathrm{~mm})$. Therefore, we could achieve up to $25 \%$ internal efficiency even when seeding the set-up with $11 \mathrm{~mJ}$. Hence, we obtained a XPW beam carrying $1.6 \mathrm{~mJ}$ pulse energy, which is the highest achieved so far to the best of our knowledge. However, we noticed that the fiber transmission was lower in this case, around $60 \%$, due to air fluctuations in the laboratory and damages caused to the fiber entrance following repeated realigning. Furthermore, at this energy level, the beam size on the crystal $(3.5 \mathrm{~mm}$ diameter) requires better crystal surface polishing quality in order to reduce the risk of damage at high intensities.

Table II summarizes the best performances achieved with each laser. One can notice that the peak-power transmission (computed as energy throughput times spectral broadening) ranges from $40 \%$ to $60 \%$. This is especially due to the accessible temporal shortening correlated to the spectral broadening experienced by the pulses. These aspects are detailed in Sec. III B along with contrast enhancement.

\section{B. Temporal quality improvement}

In this section, the temporal characterization of the XPW pulses is proposed in terms of contrast, spectral broadening, temporal shortening, and coherent contrast quality. Figure 3 compares the XPW spectrum to the input one, measured on the four laser chains. We observed the reproducibility of the XPW spectral behavior, including in each case a large broadening factor and smoothing towards Gaussian-like shape of the input spectra. These properties are already well-known and come from the nonlinear shortening of the initial pulse. In first approximation, the XPW signal intensity varies simply as the cube of the fundamental beam intensity and is reduced in duration by a factor of $\sqrt{3}$. If the pulse is transform limited, this translates to an increased spectral bandwidth by the same factor. Here, we also demonstrate that important XPW

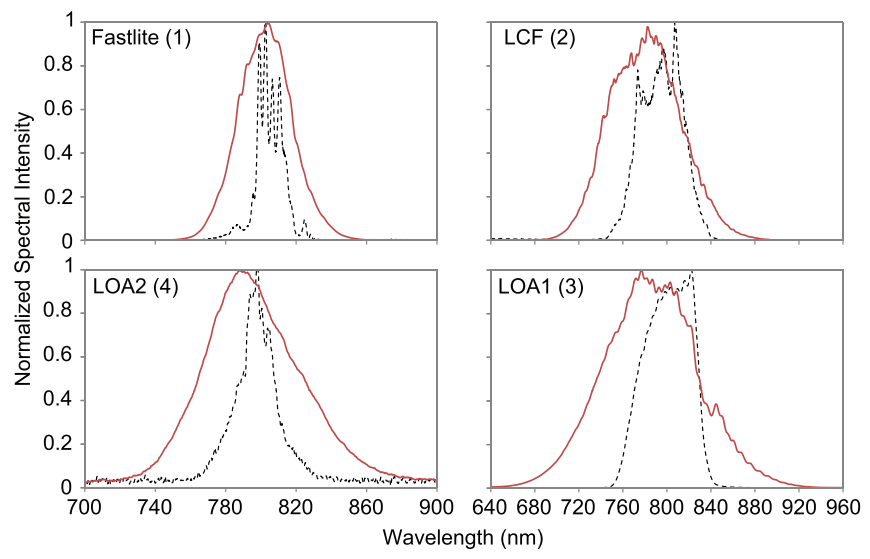

FIG. 3. Input (dotted line) and XPW (solid line) spectra for the four laser chains used.

conversion enables the generation of much shorter pulses via extra-broadening of the laser spectrum through self-phase modulation (SPM) of the fundamental pulse. The bandwidth is then transferred to the XPW pulse. However, the latter experiences a much lower SPM (factor 1/4 compared with the SPM on the fundamental). This preserves the XPW spectrum from being modulated and distorted. Broadening factors as high as 2.5 are reached when maximum XPW conversion is achieved with a low dispersive single crystal scheme, that is with a short nonlinear length $(<3 \mathrm{~mm})$. Accurate control of the initial spectral phase is absolutely necessary. A detailed study of the importance of spectral phase has been realized previously in Refs. 34, 36, and 45. The range of second-order phase within which the XPW pulse characteristics are optimal is $\pm 200 \mathrm{fs}^{2}$ at $25 \mathrm{fs}$. The same study was performed with the third-order phase, yielding a tolerance range of $\pm 2000 \mathrm{fs}^{3}$ at 25 fs. For shorter pulses, these ranges become narrower.

Compressibility of the XPW pulses has already been demonstrated. ${ }^{35}$ With the proposed set-up, we were able to compress pulses from 25 fs down to 10 fs with the LCF laser chain and from 30 fs down to 15 fs with the LOA1 laser chain using a set of appropriate chirped mirrors and glass wedges. ${ }^{45}$ Here, we also demonstrate the compression of 50 fs down to 20 fs with the Fastlite laser chain (Fig. 4(a)). In addition, using the Wizzler device, one has access to the temporal shape of the pulse over a range of a few $100 \mathrm{fs}$ around its peak. We could then confirm the sharpening of the rising edge of the pulse below -100 fs as shown in Fig. 4(b).

Finally, we confirmed the temporal contrast enhancement inherent to the XPW technique. To do so, we used the laser source LOA2, a $100 \mathrm{~Hz}$ laser system (Alpha100, Thales) delivering $11 \mathrm{~mJ}, 50 \mathrm{fs}$ at $800 \mathrm{~nm}$ pulses. We obtained $1.6 \mathrm{~mJ}$ energy pulses measured after the output polarizer from $6.5 \mathrm{~mJ}$ energy pulses before the polarizer. Taking advantage of the energetic XPW pulses, we could measure the temporal contrast with a home-made $3 \omega$-correlator over a large dynamic range. Figure 4(c) shows more than four orders of magnitude for the enhancement of the ASE level before the pulse from $10^{-4}$ to $10^{-9}$ at $-5 \mathrm{ps,} \mathrm{corresponding} \mathrm{to} \mathrm{the} \mathrm{experi-}$ mentally measured extinction ratio of the polarizers. Limits of detection are shown for both the laser and the XPW temporal intensity profile measurements at $-17.5 \mathrm{ps}$ and $-15 \mathrm{ps}$, 

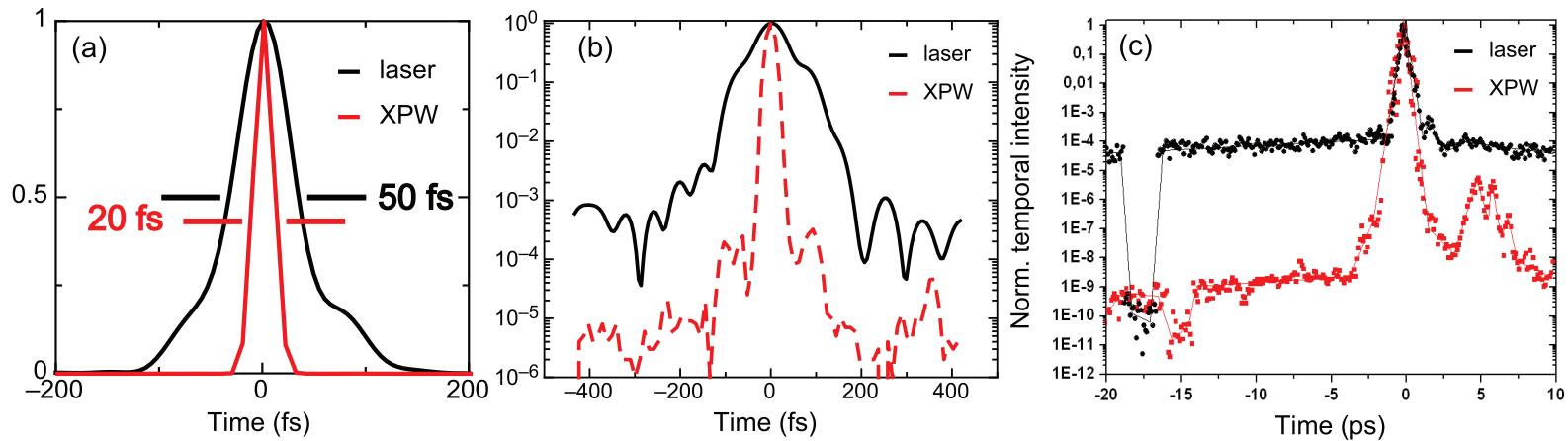

FIG. 4. Self-referenced spectral interferometry (Wizzler, Fastlite) temporal measurements of laser (1) and output XPW pulse plotted on (a) linear scale (respectively, $50 \mathrm{fs}$ and $20 \mathrm{fs}$ ) and (b) logarithmic scale (respectively, solid and dashed line); (c) $3 \omega$-autocorrelation trace of the pulse temporal profile before (dots) and after (squares) XPW for laser (4).

respectively. XPW pulses were not perfectly compressed for this measurement because the output polarizer dispersion was not compensated $\left(+500 \mathrm{fs}^{2}\right)$. Therefore, it is hard to draw conclusions from the rising edge of the pulse. In any case, the limited bandwidth of the correlator which was originally designed for longer pulses (100 fs and over) is not adapted for precise observation of ultrashort pulse features. However, the smoother shape of the spectrum along with a spectral phase improved by the XPW filtering should lead to sharp rising edge as it was shown above for laser (1). Contrast measurement with laser chains (1), (2), and (3) was not performed because either the characterization device was not available at the time or the XPW pulse energy was too low to achieve sufficiently high dynamic range. Still, the measured extinction ratio of the polarizers $\left(\approx 10^{4}\right)$ should ensure 4 orders of magnitude improvement in all the above cases (see Table II).

\section{Spatial characterization}

The cornerstone of this novel design relies on the guiding and filtering effects of the hollow-core fiber. Gaussian spatial profile propagates smoothly after the fiber without appearances of hotspots. Thus, the intensity on the crystal can be close to the continuum generation limit without inducing permanent damage to the crystal. Beam divergence and profile quality on the crystal ensure reliably optimal XPW conversion regardless of the input energy. Moreover, it provides excellent output XPW beam quality both in near-field (Fig. 5(a)) and far-field (Fig. 5(b)) with a Strehl ratio of 0.9. We observe experimentally that the XPW beam divergence is less important than that of the fundamental beam after the fiber. We attribute this property to Kerr focusing of the incident beam in the crystal. We study here the propagation of the beam after the fiber and after the crystal.

For the experiment, we use a Femtocube system from Thales Optronique SA, delivering 40 fs pulses with $0.7 \mathrm{~mJ}$ energy before the fiber. Temporal contrast is higher than $10^{3}$ at 1 ps before the main peak (measured by Wizzler, Fastlite). The laser repetition rate is $5 \mathrm{kHz}$. The energy after the fiber is $0.5 \mathrm{~mJ}$. The distance from the fiber output end to the crystal is $22.5 \mathrm{~cm}$, corresponding to a beamsize on the crystal of $1250 \mu \mathrm{m}$ (diameter at $1 / \mathrm{e}^{2}$ ). The intensity on crystal is then close to $10^{12} \mathrm{~W} / \mathrm{cm}^{2}$. We measure after the output polarizer a
XPW energy of $80 \mu \mathrm{J}$, corresponding to a global conversion efficiency of $16 \%$ and, considering reflection losses, an internal conversion efficiency close to $20 \%$. Laser fluctuations (energy, spectral phase and to a lesser extent beam profile) along with crystal quality prevented us from working closer to the damage threshold in this case. After the vacuum part of the XPW set-up, we install a $4 \mathrm{f}$ imaging system with a lens of focal length $f=225 \mathrm{~mm}$. By shifting together the lens and the CCD camera, we can then observe the XPW spatial profile (and the fundamental profile by rotating the polarizer by $90^{\circ}$ ) up to $1 \mathrm{~m}$ from the crystal surface with a magnification of 1 .

We also perform 3D numerical simulations with CommodPro (CP), ${ }^{48}$ a MIRO-based propagation code. ${ }^{49}$ MIRO was developed by the CEA for simulating laser pulse propagation in high-power laser systems. It solves the nonlinear Helmholtz equation (Eq. (2)):

$$
\Delta E-\frac{1}{c^{2}} \frac{\partial^{2} E}{\partial t^{2}}-\nabla(\nabla \cdot E)=\mu_{0} \frac{\partial^{2} P}{\partial t^{2}}
$$

where the polarization vector $\mathbf{P}$ can be expressed as a function of the electric field $\mathbf{E}$ and decomposed into two components: the linear polarization, $\mathbf{P}_{L}$ and the nonlinear polarization, $\mathbf{P}_{N L}$. The latter is simplified to Eq. (3) to account only for the first order response of centrosymetric media:

$$
P_{N L}=\left[\chi^{(3)}\right] E E^{*} E
$$

Then Miro/CP makes the assumption that the E-field can be described by a limited number of modes, $\mathbf{E}_{l}$, and therefore
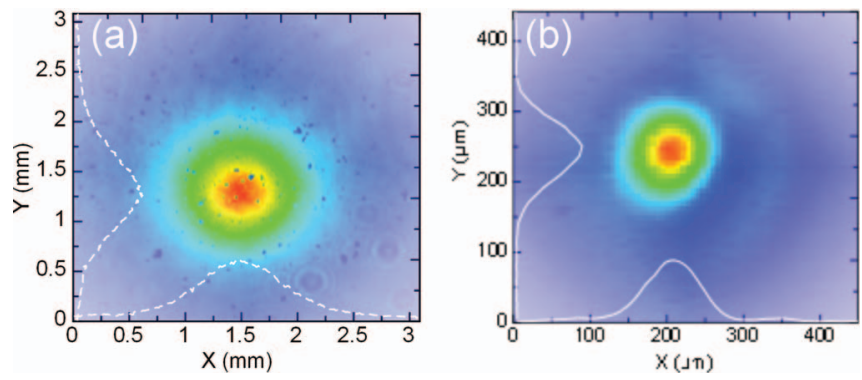

FIG. 5. Typical near-field (a) and far-field (b) XPW beam profiles measured with LOA1 laser chain. 


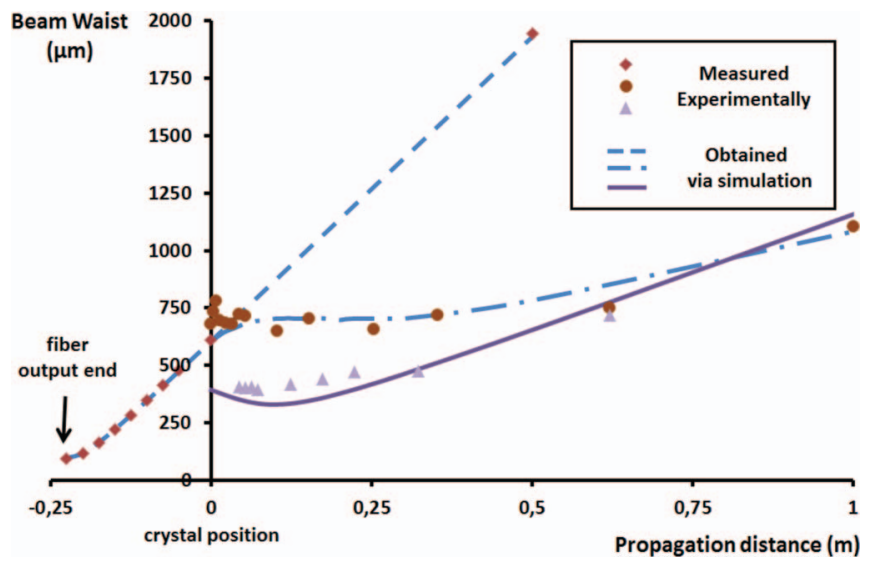

FIG. 6. Measured and simulated beam propagation from fiber output end to XPW crystal (respectively, red diamonds and dashed line), and after XPW crystal: fundamental (respectively, dots and dashed-dotted line), and XPW (respectively, triangles and solid line).

rewrites Eq. (2) as Eq. (4):

$$
\begin{aligned}
- & 2 i k_{l}\left(\frac{\partial E_{l}}{\partial z}+\frac{1}{v_{g, l}} \frac{\partial E_{l}}{\partial t}\right)+\Delta_{\perp} E_{l}+2 k_{l} \sigma_{l} \frac{\partial^{2} E}{\partial t^{2}} \\
- & i k_{l} v_{l} E_{l}+2 k_{l} \frac{\omega_{l}}{c} \frac{\gamma_{l}}{3}\left(2 E_{l} \cdot E_{l}^{*} E_{l}+E_{l} \cdot E_{l} E_{l}^{*}\right)=0,
\end{aligned}
$$

where $\Delta_{\perp}=\frac{\partial^{2}}{\partial x^{2}}+\frac{\partial^{2}}{\partial y^{2}}$ is the transverse Laplace operator, $v_{l}$ is the absorption coefficient, $\gamma_{l}$ the nonlinear index, $v_{g, l}$ $=\frac{\partial \omega}{\partial k}\left(k_{l}\right)$ is the group velocity, and $\sigma_{l}=\frac{\omega^{\prime \prime}\left(k_{l}\right)}{2 \omega^{\prime}\left(k_{l}\right)}=-\frac{1}{2} k^{\prime \prime}\left(\omega_{l}\right)$ is the group velocity dispersion.

Several calculation modes that further simplify Eq. (4) are available. However, in our case, "Phase modulation" was used as it solves the most general equation and it is well adapted for ultrashort pulses. CP was then specifically modified to include the XPW generation process. In particular, an element called "XPW crystal" was added to the plate element section. This element includes specific parameters necessary for XPW generation, such as crystallographic orientation (with option for [001]- and [011]-orientation), $\chi^{(3)}$, and $\sigma$ in addition to the usual parameters available. Therefore, when using the "XPW crystal" element, a linearly polarized input pulse generates a cross-polarized wave. The $\mathrm{CP}$ interface then allows the observation of fundamental and XPW beam characteristics, separately. Correct definition of the process in the $\mathrm{CP}$ software was checked by comparing efficiency curve, crystal orientation dependence, and spectral behaviour with experimental data and with another validated home-made 1D split-step propagation code. In addition to the complete spatio-temporal definition of the input pulse, CP allows detailed observation of spatial propagation.

Figure 6 summarizes the experimental and numerical results: beam waist at $1 / \mathrm{e}^{2}$ from the fiber to the crystal position and then up to $1 \mathrm{~m}$ after the crystal. The full beam divergence after the fiber is $5.2 \mathrm{mrad}$ with an output beam waist of $96 \mu \mathrm{m}$. We first check that the beam size on the crystal is what we expect given the fiber inner diameter. The measured beam diameter (at $1 / \mathrm{e}^{2}$ ) is on the order of $1250 \mu \mathrm{m}$. It corresponds to an intensity on crystal of $0.9 \times 10^{12} \mathrm{~W} / \mathrm{cm}^{2}$. This value is below the maximum intensity normally achievable with $\mathrm{BaF}_{2}$ crystals (a few $10^{12} \mathrm{~W} / \mathrm{cm}^{2}$ ) and also consistent with the efficiency observed (20\% internally), inferior to $30 \%-35 \%$ normally accessible. From Fig. 6 , it is visible that the XPW beamsize is a factor of 1.55 smaller than the fundamental beam size at the crystal output (waists of $450 \mu \mathrm{m}$ and $700 \mu \mathrm{m}$, respectively). This is in agreement with the theoretical factor of $\sqrt{3}$ corresponding to a third-order nonlinear effect. The divergence of the beam is strongly reduced after propagation in the crystal due to Kerr-lensing. Furthermore, both XPW and fundamental beams slightly focus after the crystal and then diverge at different speeds. The feature is, however, less pronounced than in the case of an input beam focused on the crystal. ${ }^{38,50}$ The fact that the fundamental
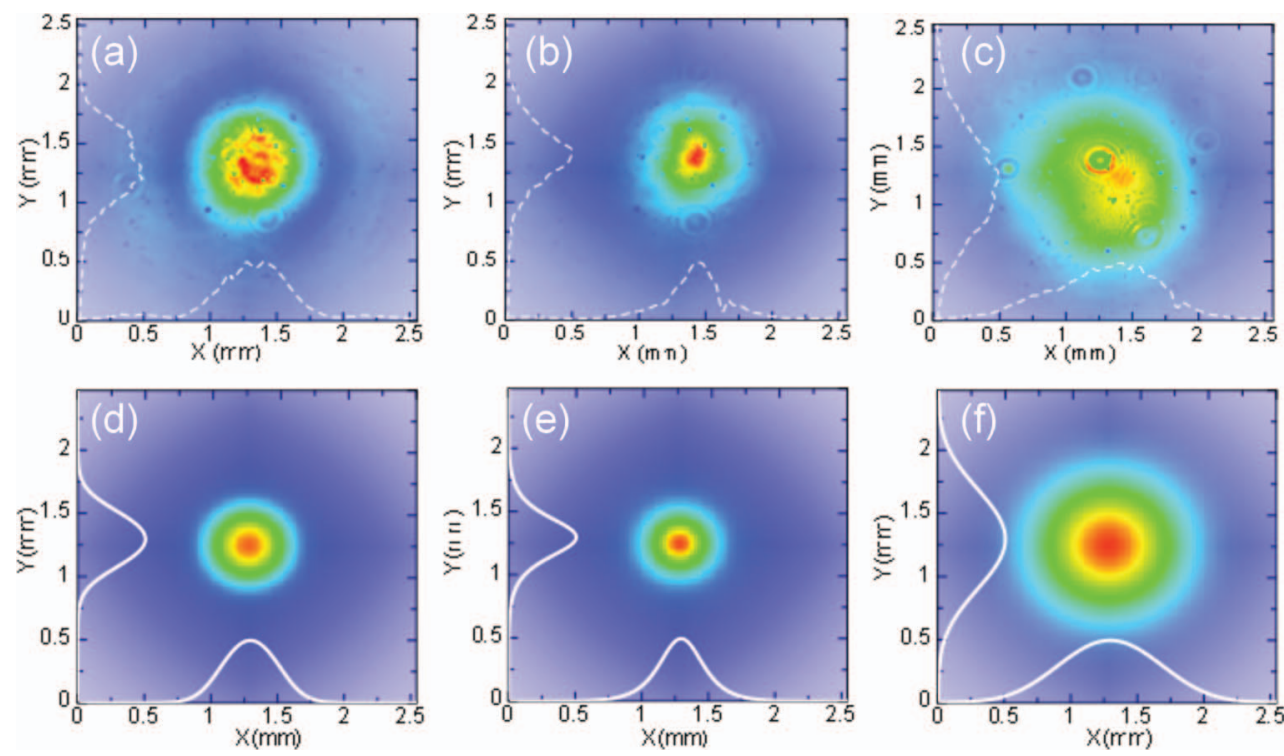

FIG. 7. Measured (top row) and simulated (bottom row) XPW beam profiles at the crystal position (respectively, (a) and (d)), at the refocusing position ( $\approx 7 \mathrm{~cm}$ away from the crystal) (respectively, (b) and (e)) and $65 \mathrm{~cm}$ after the crystal (respectively, (c) and (f)). Beam profiles were measured with Thales Optronique Femtocube laser source and simulated ones were obtained with CommodPro software. 
beam does undergo strong Kerr-lensing but no strong focusing prevents early saturation of the XPW process, due to accumulated nonlinear phase, usually observed in a single-crystal scheme ${ }^{26,40}$ The spatial propagation experimentally observed is well reproduced by numerical simulation. $\mathrm{CP}$ enables to point out the critical influence of the initial divergence on the beam propagation after the nonlinear crystal, opening the way to adapt the fiber inner diameter to get specific propagation such as collimated XPW beam.

Figure 7 shows the XPW beam profiles at different locations. Both measured and simulated profiles are displayed. Figure 7(a) shows the XPW pulse at the crystal output. Its ellipticity of $95 \%$ highlights the spatial filtering effect of the fiber. Figure 7(b) shows the profile where the XPW beam is focused by Kerr effect. The profile is still symmetric but it exhibits a slightly leptokurtic shape. However after some propagation $(65 \mathrm{~cm}$ as shown in Fig. 7(c)), the profile recovers a Gaussian-like shape. As a comparison, we plotted simulated profiles from identical input beam characteristics on the crystal and identical interaction conditions (energy, pulse duration, efficiency). Figures 7(d)-7(f) summarizes the results taken at the same locations as the measured profiles: crystal output, $7 \mathrm{~cm}$ and $65 \mathrm{~cm}$ after. It demonstrates the good agreement between the experimental and numerical data. In particular, the beam refocusing position is well determined and the beam reshaping is similarly observed with propagation.

\section{CONCLUSION}

We presented the complete description and characterization of a novel set-up design allowing energy-scalable XPW generation for contrast enhancement of femtosecond laser pulses. By filtering the input beam through a hollow-core fiber, we are able to achieve nearly maximum theoretical internal conversion (35\%) for input pulse energies ranging from hundreds of microjoules to more than $10 \mathrm{~mJ}$. Large spectral broadening factors $(>2)$ are obtained, corresponding to strong pulse shortening. Moreover, thanks to fiber spatial filtering, the XPW pulse propagates over long distances with excellent beam profile quality. Global efficiency of the order of $20 \%$ and temporal shortening by a factor larger than 2 lead to a peak-power transmission of the filter of the order of 50\%. For example, using an $11 \mathrm{~mJ}, 40 \mathrm{fs}$ input at $100 \mathrm{~Hz}$ repetition rate, $1.6 \mathrm{~mJ}, 15$ fs Fourier-transform limit pulses were obtained. It corresponds to a global energy transmission of $14.5 \%$ and a peak-power transmission of $40 \%$. At this energy level, such efficiencies suggest that the XPW filter could be used at the end of a CPA chain as a post compressor.

\section{ACKNOWLEDGMENTS}

Financial support from the Agence Nationale pour la Recherche (Grant No. ANR-09-JCJC-0063) and from RTRA - Triangle de la Physique through program 2011-024T - NewXPW is gratefully acknowledged.

${ }^{1}$ J. P. Freidberg, R. W. Mitchell, R. L. Morse, and L. I. Rudsinski, Phys. Rev. Lett. 28, 795-799 (1972).

${ }^{2}$ F. Brunel, Phys. Rev. Lett. 59, 52-55 (1987).
${ }^{3}$ D. Neely, P. Foster, A. Robinson, F. Lindau, O. Lundh, A. Persson, C.-G. Wahlstrom, and P. McKenna, Appl. Phys. Lett. 89, 021502 (2006).

${ }^{4}$ T. Ceccotti, A. Lévy, H. Popescu, F. Réau, P. D’Oliveira, P. Monot, J. P. Geindre, E. Lefebvre, and P. Martin, Phys. Rev. Lett. 99, 185002 (2007).

${ }^{5}$ A. Flacco, F. Sylla, M. Veltcheva, M. Carrié, R. Nuter, E. Lefebvre, D. Batani, and V. Malka, Phys. Rev. E 81, 036405 (2010).

${ }^{6}$ J. Itatani, J. Faure, M. Nantel, G. Mourou, and S. Watanabe, Opt. Commun. 148, 70-74 (1998).

${ }^{7}$ M. Aoyama, T. Harimoto, J. Ma, Y. Akahane, and K. Yamakawa, Opt. Express 9, 579-585 (2001).

${ }^{8}$ A. Marcinkevicius, R. Tommasini, G. D. Tsakiris, K. J. Witte, E. Gaizauskas, and U. Teubner, Appl. Phys. B 79, 547-554 (2004).

${ }^{9}$ A. Renault, F. Augé-Rochereau, T. Planchon, P. d'Oliveira, T. Auguste, G. Chériaux, and J. P. Chambaret, Opt. Commun. 248, 535-541 (2005).

${ }^{10}$ A. Dubietis, G. Jonusauskas, and A. Piskarskas, Opt. Commun. 88, 437440 (1992).

${ }^{11}$ H. Yoshida, E. Ishii, R. Kodama, H. Fujita, Y. Kitagawa, Y. Izawa, and T. Yamanaka, Opt. Lett. 28, 257-259 (2003).

${ }^{12}$ C. Dorrer, I. Begishev, A. Okishev, and J. Zuegel, Opt. Lett. 32, 2143-2145 (2007).

${ }^{13}$ H. Kiriyama, M. Mori, Y. Nakai, T. Shimomura, M. Tanoue, A. Akutsu, H. Okada, T. Motomura, S. Kondo, S. Kanazawa, A. Sagisaka, J. Ma, I. Daito, H. Kotaki, H. Daido, S. Bulanov, T. Kimura, and T. Tajima, Opt. Commun. 282, 625-628 (2009).

${ }^{14}$ H. Kapteyn, M. Murnane, A. Szoke, and R. Falcone, Opt. Lett. 16, 490-492 (1991).

${ }^{15}$ S. Backus, H. Kapteyn, M. Murnane, D. Gold, H. Nathel, and W. White, Opt. Lett. 18, 134-136 (1993).

${ }^{16}$ G. Doumy, F. Quéré, O. Gobert, M. Perdrix, P. Martin, P. Audebert, J. C. Gauthier, J.-P. Geindre, and T. Wittmann, Phys. Rev. E 69, 026402 (2004).

${ }^{17}$ A. Jullien, O. Albert, F. Burgy, G. Hamoniaux, J. P. Rousseau, J. Chambaret, F. Augé-Rochereau, G. Chériaux, J. Etchepare, N. Minkovski, and S. Saltiel, Opt. Lett. 30, 920-922 (2005).

${ }^{18}$ A. Borot, A. Malvache, X. Chen, D. Douillet, G. Iaquianiello, T. Lefrou, P. Audebert, J. Geindre, G. Mourou, F. Quéré, and R. Lopez-Martens, Opt. Lett. 36, 1461-1463 (2011).

${ }^{19}$ A. I. Kovrigin, D. V. Yakovlev, B. V. Zhdanov, and N. I. Zheludev, Opt. Commun. 35, 92 (1980).

${ }^{20}$ M. G. Dubenskaya, R. S. Zadoyan, and N. I. Zheludev, J. Opt. Soc. Am. B 2, 1174-1178 (1985).

${ }^{21}$ D. Hutchings, J. Aitchison, B. Wherrett, G. Kennedy, and W. Sibbett, Opt. Lett. 20, 991-993 (1995).

${ }^{22}$ D. Hutchings, J. Aitchison, and J. Arnold, J. Opt. Soc. Am. B 14, 869-879 (1997).

${ }^{23}$ G. Petrov, O. Albert, J. Etchepare, and S. Saltiel, Opt. Lett. 26, 355-357 (2001).

${ }^{24}$ N. Minkovski, S. Saltiel, G. Petrov, O. Albert, and J. Etchepare, Opt. Lett. 27, 2025-2027 (2002).

${ }^{25}$ N. Minkovski, G. Petrov, S. Saltiel, O. Albert, and J. Etchepare, J. Opt. Soc. Am. B 21, 1659-1664 (2004).

${ }^{26}$ L. Canova, S. Kourtev, N. Minkovski, A. Jullien, R. Lopez-Martens, O. Albert, and S. M. Saltiel, Appl. Phys. Lett. 92, 231102 (2008).

${ }^{27}$ M. Kalashnikov, E. Risse, H. Schonnagel, and W. Sandner, Opt. Lett. 30, 923-925 (2005).

${ }^{28}$ V. Chvykov, P. Rousseau, S. Reed, G. Kalinchenko, and V. Yanovsky, Opt. Lett. 31, 1456-1458 (2006).

${ }^{29}$ M. Kalashnikov, K. Osvay, R. Volkov, H. Schonnagel, and W. Sandner, in CLEO:2011 - Laser Applications to Photonic Applications, OSA Technical Digest (CD) (Optical Society of America, 2011), paper CWG3.

${ }^{30}$ J. Mikhailova, A. Buck, A. Borot, K. Schmid, C. Sears, G. Tsakiris, F. Krausz, and L. Veisz, Opt. Lett. 36, 3145-3147 (2011).

${ }^{31}$ F. Lureau, S. Laux, O. Casagrande, C. Radier, O. Chalus, F. Caradec, and C. Simon-Boisson, Proc. SPIE 8235, 823513 (2012).

${ }^{32}$ See http://www.thalesgroup.com for information on available XPW setups.

${ }^{33}$ See http://www.amplitude-technologies.com information on available XPW set-ups.

${ }^{34}$ L. Canova, O. Albert, N. Forget, B. Mercier, S. Kourtev, N. Minkovski, S. M. Saltiel, and R. Lopez-Martens, Appl. Phys. B 93, 443-453 (2008).

${ }^{35}$ A. Jullien, J. P. Rousseau, B. Mercier, L. Antonucci, O. Albert, G. Chériaux, S. Kourtev, N. Minkovski, and S. M. Saltiel, Opt. Lett. 33, 23532355 (2008). 
${ }^{36}$ A. Jullien, X. Chen, A. Ricci, J. P. Rousseau, R. Lopez-Martens, L. Ramirez, D. Papadopoulos, A. Pellegrina, F. Druon, and P. Georges, Appl. Phys. B 102, 769-774 (2011).

${ }^{37}$ T. Oksenhendler, S. Coudreau, N. Forget, V. Crozatier, S. Grabielle, R. Herzog, O. Gobert, and D. Kaplan, Appl. Phys. B 99, 7-12 (2010).

${ }^{38}$ D. E. Adams, T. A. Planchon, J. A. Squier, and C. G. Durfee, Opt. Lett. 35, 1115-1117 (2010)

${ }^{39}$ Y. Zaouter, L. P. Ramirez, D. N. Papadopoulos, C. Honninger, M. Hanna, F. Druon, E. Mottay, and P. Georges, Opt. Lett. 36, 1830-1832 (2011).

${ }^{40}$ A. Jullien, O. Albert, G. Chériaux, J. Etchepare, S. Kourtev, N. Minkovski, and S. M. Saltiel, Opt. Express 14, 2760-2769 (2006).

${ }^{41}$ P. M. Paul and L. Canova, "Development of an 10-14 ultra high contrast laser system using high energy XPW filtering scheme," Conference on Lasers and Electro-Optics (CLEO) and Quantum Electronics and Laser Science Conference (QELS), 16-21 May 2010, pp. 1 and 2.

${ }^{42}$ E. A. J. Marcatili and R. A. Schmeltzer, Bell Syst. Tech. J. 43, 1783-1809 (1964).
${ }^{43}$ M. Nisoli, S. De Silvestri, and O. Svelto, Appl. Phys. Lett. 68, 2793-2795 (1996).

${ }^{44}$ M. Nisoli, S. De Silvestri, O. Svelto, R. Szipocs, K. Ferencz, C. Spielmann, S. Sartania, and F. Krausz, Opt. Lett. 22, 522-524 (1997).

${ }^{45}$ L. P. Ramirez, D. N. Papadopoulos, A. Pellegrina, P. Georges, F. Druon, P. Monot, A. Ricci, A. Jullien, X. Chen, J. P. Rousseau, and R. LopezMartens, Opt. Express 19, 93-98 (2011).

${ }^{46}$ D. Marcuse, J. Opt. Soc. Am. 68, 103-109 (1978).

${ }^{47}$ X. Chen, L. Canova, A. Malvache, A. Jullien, R. Lopez-Martens, C. G. Durfee, D. N. Papadopoulos, and F. Druon, Appl. Phys. B 99, 149-157 (2010).

${ }^{48}$ See www.oxalis-laser.com for more information on CommodPro software. ${ }^{49}$ O. Morice, Opt. Eng. 42, 1530-1541 (2003).

${ }^{50}$ C. G. Durfee, L. Canova, X. Chen, A. Trisorio, O. Albert, and R. Lopez-Martens, "Spatio-Temporal Dynamics in Efficient Double-Crystal Cross Polarized Wave Generation," in Conference on Lasers and ElectroOptics/International Quantum Electronics Conference, OSA Technical Digest (CD) (Optical Society of America, 2009), paper CFC1. 